\title{
Assessment of functional tricuspid regurgitation using 320-detector-row multislice computed tomography: Risk factor analysis for recurrent regurgitation after tricuspid annuloplasty
}

\author{
Masashi Kabasawa, MD, ${ }^{\text {a }}$ Hiroki Kohno, MD, PhD, ${ }^{\text {a }}$ Toru Ishizaka, MD, PhD, ${ }^{a}$ Keiichi Ishida, MD, PhD, ${ }^{a}$ \\ Nobusada Funabashi, $\mathrm{MD}, \mathrm{PhD},{ }^{\mathrm{b}}$ Akihisa Kataoka, $\mathrm{MD}, \mathrm{PhD},{ }^{\mathrm{b}}$ and Goro Matsumiya, $\mathrm{MD}, \mathrm{PhD}^{\mathrm{a}}$
}

\begin{abstract}
Objective: Functional tricuspid regurgitation (TR) often develops secondary to left heart disease. Tricuspid annuloplasty (TAP) is usually the treatment of choice for significant TRs, but recurrence of TR after surgery can occur. Previous studies have not clearly demonstrated the cause of the recurrent TR after TAP. By using an electrocardiogram-gated 320-detector-row multislice computed tomography (CT), we sought to delineate the morphologic cause of the incompetent tricuspid valve and identify the risk factors for recurrent TR.
\end{abstract}

\begin{abstract}
Methods: From August 2010 to September 2011, 35 patients underwent preoperative CT of the tricuspid valve. The distance between each commissure, the tethering angle of each leaflet, and the tethering height were measured. TAP using a rigid annuloplasty ring was performed in 22 patients. Risk factors for recurrent TR were determined by multivariate analyses.
\end{abstract}

Results: End-diastolic and end-systolic tricuspid valve annular diameters (TVAD) correlated significantly with preoperative TR severity $\left(R^{2}, 0.2734-0.4287 ; P<.05\right)$. However, compared with TVAD, tethering angles and height showed stronger correlation with preoperative TR severity $\left(R^{2}\right.$ : tethering angles, $0.5769-0.6810$; tethering height, 0.6854). Multivariate analysis revealed that tethering height was an independent risk factor of postoperative recurrent TR $(P=.0069)$.

Conclusions: TVAD, tethering angles, and tethering height correlated significantly with preoperative TR severity. The tethering height of the tricuspid valve showed significant correlation with recurrent TR. (J Thorac Cardiovasc Surg 2014;147:312-20)

Functional tricuspid regurgitation (TR) mainly occurs from tricuspid annular dilation and right ventricular enlargement, which often develop secondary to left heart failure from myocardial or valvular causes, right ventricular volume and pressure overload, and subsequent dilation of the cardiac chambers. ${ }^{1,2}$ Several reports suggest that an increase in TR severity is associated with worse prognosis or worse quality of life., ${ }^{1,3}$ Tricuspid annuloplasty (TAP), including De Vega, Kay-Reed, and implantation of a flexible or rigid ring, is usually the treatment of choice for significant TR, although reports of recurrent TR after the procedure (up to $61 \%$ recurrence rate) exist. ${ }^{4-8}$ Despite the occurrence of postoperative $\mathrm{TR}$, reoperation is rarely performed ( $3 \%$ at 8 years) inasmuch as operative risks are usually considered high, reaching 37\% in-hospital mortality in some cases. ${ }^{5}$ Low left ventricular (LV) ejection fraction, atrial fibrillation, and pulmonary hypertension

From the Departments of Cardiovascular Surgery, ${ }^{\mathrm{a}}$ and Cardiovascular Science and Medicine, ${ }^{\mathrm{b}}$ Chiba University Graduate of Medicine, Chiba, Japan.

Disclosures: Authors have nothing to disclose with regard to commercial support.

Received for publication Dec 18, 2011; revisions received June 15, 2012; accepted for publication Nov 6, 2012; available ahead of print Dec 14, 2012.

Address for reprints: Goro Matsumiya, MD, PhD, Department of Cardiovascular Surgery, Chiba University Graduate School of Medicine, 1-8-1 Inohana, Chuo-ku, Chiba City, Chiba 260-8670, Japan (E-mail: matsumg@faculty.chiba-u.jp). $0022-5223 / \$ 36.00$

Copyright (c) 2014 by The American Association for Thoracic Surgery http://dx.doi.org/10.1016/j.jtcvs.2012.11.017 have all been reported as independent risk factors for postoperative recurrent TR. ${ }^{5,6}$

Very few studies have correlated the morphology of the incompetent tricuspid valve with the risk of recurrent TR. $^{6,7}$ Those who have examined the morphologic pathogenesis of recurrent TR have used a 2-dimensional transthoracic echocardiogram (TTE), but technical problems relating to reproducibility of the images and accurate delineation of the tricuspid valve anatomy have limited its use. In recent years, real-time 3-dimensional echocardiograms have gained popularity inasmuch as they complement the weakness of 2-dimensional imaging. ${ }^{9-13}$ However, a similar technical limitation persists; the right heart is still difficult to fully visualize. A promising alternative to echocardiograms is multislice computed tomography (CT), which provides more detailed and reproducible geometric measurements and 4-dimensional delineation of the right heart. In this study, we souht to demonstrate the cause of functional TR from a morphologic aspect using an electrocardiogram (ECG)-gated 320-detector-row multislice $\mathrm{CT}$ and identify the risk factors for recurrent TR after TAP.

\section{PATIENTS AND METHODS}

Study Population

From August 2010 to September 2011, ECG-gated 320-detector-row CT was performed in 35 patients. Patient characteristics are shown in 


\begin{tabular}{|c|c|}
\hline \multicolumn{2}{|c|}{ Abbreviations and Acronyms } \\
\hline A & $=$ middle of anterior leaflet annulus \\
\hline $\mathrm{A} \alpha$ & $=$ tethering angle of anterior leaflet \\
\hline AP & $=$ anteroposterior commissure \\
\hline AS & $=$ anteroseptal commissure \\
\hline CT & $=$ computed tomography \\
\hline dTVAD & $\begin{aligned}= & \text { end-diastolic tricuspid valve } \\
& \text { annular diameter }\end{aligned}$ \\
\hline dTVAD(AS-A & $\begin{aligned}= & \text { end-diastolic tricuspid valve } \\
& \text { annular diameter between } \\
& \text { anteroseptal commissure and } \\
& \text { anteroposterior commissure }\end{aligned}$ \\
\hline dTVAD(AP-SP) & $\begin{array}{l}=\text { end-diastolic tricuspid valve } \\
\text { annular diameter between } \\
\text { anteroposterior commissure and } \\
\text { septoposterior commissure }\end{array}$ \\
\hline dTVAD(TTE) & $\begin{aligned}= & \text { end-diastolic tricuspid valve } \\
& \text { annular diameter on } \\
& \text { transthoracic echocardiogram }\end{aligned}$ \\
\hline ECG & $=$ electrocardiogram \\
\hline LV & $=$ left ventricular \\
\hline LVDd & $\begin{aligned}= & \text { left ventricular diastolic } \\
& \text { diameter }\end{aligned}$ \\
\hline LVDs & $=$ left ventricular systolic diameter \\
\hline MR & $=$ mitral regurgitation \\
\hline $\mathrm{P}$ & $\begin{aligned} &= \text { middle of posterior leaflet } \\
& \text { annulus }\end{aligned}$ \\
\hline $\mathrm{P} \alpha$ & $\begin{aligned}= & \text { tethering angle of posterior } \\
& \text { leaflet }\end{aligned}$ \\
\hline $\mathrm{S}$ & $=$ middle of septal leaflet annulus \\
\hline $\mathrm{S} \alpha$ & $=$ tethering angle of septal leaflet \\
\hline SP & $=$ septoposterior commissure \\
\hline sTVAD & $\begin{aligned}= & \text { end-systolic tricuspid valve } \\
& \text { annular diameter }\end{aligned}$ \\
\hline TAP & $=$ tricuspid annuloplasty \\
\hline TR & $=$ tricuspid regurgitation \\
\hline $\begin{array}{l}\text { TR jet area/ } \\
\text { RA area }\end{array}$ & $\begin{array}{l}=\mathrm{TR} \text { jet area over the area of } \\
\text { the right atrium }\end{array}$ \\
\hline TRPG & $\begin{array}{l}=\text { tricuspid regurgitation pressure } \\
\text { gradient }\end{array}$ \\
\hline TTE & $=$ transthoracic echocardiogram \\
\hline TVA & $=$ tricuspid valve annular diameter \\
\hline TVAD ( & $\begin{aligned}= & \text { tricuspid valve annular diameter } \\
& \text { measured during surgery }\end{aligned}$ \\
\hline
\end{tabular}

Table 1. Patients with chronic renal failure (serum creatinine $<2.0 \mathrm{mg} / \mathrm{dL}$ or estimated glomerular filtration rate $<30 \mathrm{~mL} \cdot \min ^{-1} \cdot 1.73 \mathrm{~m}^{-2}$ ) and emergency operations were excluded from the study. Informed consent was obtained from all patients before the $\mathrm{CT}$ was performed.

TAP was performed in 22 patients among the aforementioned 35 patients. Characteristics of these 22 patients and operative data are shown in Table 2. The indications for TAP were as follows: (1) greater than mild TR and (2) preoperative tricuspid annular diameter of greater than $40 \mathrm{~mm}$ on CT or TTE. Patients in whom degenerative mitral regurgitation (MR) or functional MR (ischemic or LV enlargement owing to sarcoidosis) was diagnosed had severe regurgitation and all received mitral valve repair with annuloplasty. Mitral valve replacement was performed in 6 patients with rheumatic mitral stenosis and 1 patient with infective endocarditis.

\section{Protocol for ECG-Gated 320-Detector-Row Multislice CT}

The ECG-gated 320-detector-row multislice CT (Aquilion one, Toshiba Medial Systems, Tokyo, Japan) was programmed to have a slice thickness of $0.5 \mathrm{~mm}$. A HeartNAVI system (Toshiba Medical Systems), which provides temporal resolution that can be optimized according to the patient's heart rate, was used for this study. The following algorithm, based on heart rate, was used to reconstruct the images: less than 66 beats/min, half reconstruction from a heartbeat; 66 to 79 beats/min, 2-sector reconstruction from 2 heartbeats; greater than 80 beats/min, 3-sector reconstruction from 3 beats. Additional data were acquired from 1 extra heartbeat in excess of earlier estimates if unexpected heartbeat irregularities were observed.

We developed a novel biphasic protocol for contrast agent injection to conduct this study. During the first phase, $50 \mathrm{~mL}$ of undiluted contrast agent was injected at $2.5 \mathrm{~mL} / \mathrm{s}$, followed by $90 \mathrm{~mL}$ of a $50 \% / 50 \%$ saline-tocontrast agent mixture at $5.5 \mathrm{~mL} / \mathrm{s}$. Scanning was initiated on completion of the injection. For patients with atrial fibrillation, the maximum R-to- $\mathrm{R}$ interval was selected to perform half reconstruction of the images. After scanning, images were reconstructed every $5 \%$ from $0 \%$ to $95 \%$ of the $\mathrm{R}$-to-R interval, and the reconstructed volume data images were transferred to a workstation (ZIOSTATION; Ziosoft, Tokyo, Japan). This workstation was used for 4-dimensional reconstruction of images and measurement of the valve geometry.

We defined the middle of the anterior leaflet annulus as A, the middle of the septal leaflet annulus as S, the middle of the posterior leaflet annulus as $\mathrm{P}$, the anteroseptal commissure as AS, the anteroposterior commissure as AP, and the septoposterior commissure as SP (Figure 1, A). We defined the intercommissural distance as tricuspid valve annular diameter (TVAD). The end-diastolic TVAD (dTVAD) and end-systolic TVAD (sTVAD) between AS and AP (AS-AP), AS and SP (AS-SP), and AP and SP (AP-SP) were measured (Figure 1, B). The angles between the annular plane and each leaflet measured at $\mathrm{A}, \mathrm{S}$, and $\mathrm{P}$ at end-systolic phase were defined as $\mathrm{A} \alpha, \mathrm{S} \alpha$, andP $\alpha$, respectively (tethering angles) (Figure 1, $C$ ). The maximum distance between the point of coaptation of the leaflets and the annular plane (defined as the plane containing AS, AP, and SP) was also measured (tethering height) (Figure 1,E).

\section{Transthoracic Echocardiogram}

TTE was performed using Phillips IE-33 (Phillips Medical Systems, Andover, Mass), Aplio SSA-770A (Toshiba Medical Systems), or Acuson Sequoia 512 (Siemens Medical Solutions, Mountainview, Calif) ultrasonography systems. The severity of preoperative TR was classified on the basis of the calculation of TR jet area over the area of the right atrium (TR jet area/RA area) from data acquired from the TTE 4-chamber view $^{3,6,11}$ : TR0 as none $(0)$; TR1 as trivial $(0-0.05)$; TR2 as mild $(0.05-0.2)$; TR3 as moderate $(0.2-0.5)$; and TR4 as severe $(0.5-1)$. The dTVAD(TTE) was also obtained from the 4-chamber view. Additionally, TTE was performed 1 week after TAP, and the severity of postoperative TR was assessed similarly.

\section{Operations}

A median sternotomy was performed, and mild hypothermic cardiopulmonary bypass was established. Under intermittent cold blood cardioplegia, an incision was made in the right atrium and the tricuspid valve was exposed. Stitches were placed along the annulus of the tricuspid valve 
TABLE 1. Patient characteristics

\begin{tabular}{lc}
\hline \multicolumn{1}{c}{$\mathbf{n}$} & $\mathbf{3 5}$ \\
\hline Age $(\mathrm{y})$ & $65.2 \pm 11.0(38-81)$ \\
Sex (male/female) & $19: 16$ \\
Af & 19 \\
PH (mPAP $>25 \mathrm{~mm} \mathrm{Hg})$ & 14 \\
Low LVEF $(>35 \%)$ & 3 \\
Diagnosis & \\
AS & 5 \\
AR & 6 \\
MS & 6 \\
MR & 21 \\
ASD & 4 \\
IHD & 7 \\
Preoperative TR severity & \\
0 (none) TR jet/RA area $=0$ & 2 \\
1 (trivial) 0 TR jet/RA area $\leq 0.05$ & 4 \\
2 (mild) 0.05 TR jet/RA area $\leq 0.2$ & 14 \\
3 (moderate) 0.2 TR jet/RA area $\leq 0.5$ & 7 \\
4 (severe) 0.5 TR jet/RA area & 8 \\
TAP & \\
+ &
\end{tabular}

$A f$, Atrial fibrillation; $P H$, pulmonary hypertension; $P A P$, pulmonary arterial pressure; $L V E F$, left ventricular ejection fraction; $A S$, aortic stenosis; $A R$, aortic regurgitation; $M S$, mitral stenosis; $M R$, mitral regurgitation; $A S D$, atrial septal defect; $I H D$, ischemic heart disease; $T R$, tricuspid regurgitation; $T R$ jet area/RA area, TR jet area over the area of the right atrium; TAP, tricuspid annuloplasty.

clockwise from the anteroseptal commissure to the middle of the septal leaflet. TAP was performed using Edwards $\mathrm{MC}^{3}$ tricuspid annuloplasty rings (Edwards Lifesciences, Irvine, Calif). A ring size of $28 \mathrm{~mm}$ was

TABLE 2. Characteristics of patients who underwent TAP

\begin{tabular}{|c|c|}
\hline $\mathbf{n}$ & 22 \\
\hline Age & $67.3 \pm 9.2$ y $(48-81)$ \\
\hline Sex (male/female) & $12: 10$ \\
\hline Af & 16 \\
\hline LVDd (mm) & $53.5 \pm 10.3$ \\
\hline LVDs (mm) & $37.6 \pm 9.7$ \\
\hline MR severity & $2.6 \pm 1.2$ \\
\hline TRPG (mm Hg) & $38.1 \pm 12.0$ \\
\hline $\mathrm{PH}(\mathrm{mPAP}>25 \mathrm{~mm} \mathrm{Hg})$ & 12 \\
\hline \multicolumn{2}{|l|}{$\mathrm{MC}^{3}$ ring size } \\
\hline $28 \mathrm{~mm}$ & 21 \\
\hline $26 \mathrm{~mm}$ & 1 \\
\hline \multicolumn{2}{|l|}{ Concomitant procedure } \\
\hline AVR & 5 \\
\hline MVR & 7 \\
\hline MVP & 7 \\
\hline MAP & 3 \\
\hline ASD closure & 3 \\
\hline CABG & 2 \\
\hline
\end{tabular}

$T A P$, Tricuspid annuloplasty; $A f$, atrial fibrillation; $L V D d$, left ventricular diastolic diameter; $L V D s$, left ventricular systolic diameter; $M R$, mitral regurgitation; TRPG, tricuspid regurgitation pressure gradient; $P H$, pulmonary hypertension; $m P A P$, mean pulmonary artery pressure; $A V R$, aortic valve replacement; $M V R$, mitral valve replacement; $M V P$, mitral valvuloplasty; $M A P$, mitral annuloplasty; $A S D$, atrial septal defect; $C A B G$, coronary artery bypass grafting. selected for all patients, with the exception of 1 case in which a 26-mm ring was used because of small body size. The distance between the anteroseptal commissure and the anteroposterior commissure (TVAD[op]) was directly measured with a sterile flexible ruler before the implantation of the prosthetic tricuspid ring (Figure 1,D).

\section{Statistical Analysis}

For the baseline variables, summary statistics were constructed using frequencies and proportions for categorical data and standard deviations for continuous variables. The effects of preoperative CT morphologic parameters on preoperative and postoperative TR jet area/RA area were estimated by a linear regression analysis, and the goodness of fit of the model was checked by the determination coefficient $\left(R^{2}\right)$. Multivariate linear regression analyses were performed with TR jet area/RA area after TAP as the dependent variable to adjust the effects of relevant covariates. Additionally, risk factors for recurrent TR2 or more were evaluated by logistic regression analysis. One criterion for evaluating the cutoff point was to maximize the sum of sensitivity and specificity. All comparisons were planned and the tests were 2-sided. All statistical analyses were performed using the SAS software program, version 9.2, and JMP, version 9.0.0 (SAS Institute Inc, Cary, NC).

\section{RESULTS}

To validate the accuracy of the tricuspid valve measurements on $\mathrm{CT}$, we performed a preliminary study that compared the correlation between TVAD(op) and dTVAD(AS-AP) on CT and that between TVAD(op) and dTVAD(TTE) in 22 patients undergoing TAP (Figure 2). dTVAD(AS-AP) on CT correlated more strongly with TVAD(op) than dTVAD(TTE) with TVAD(op) $\left(R^{2}\right.$ : CT 0.8761 vs TTE 0.6170 ).

We then studied the correlation between tricuspid valve geometry and preoperative TR severity in all 35 patients, including those in whom TAP was not indicated. TVAD on CT correlated with preoperative TR severity ( $R^{2}$ : dTVAD(AS-AP), 0.3432; dTVAD(AS-SP), 0.3169; dTVAD(AP-SP), $0.4287 ; P<.05$; Figure $3, A$ to $C$ ). sTVAD on CT also correlated with preoperative TR severity $(P<.05$ for all intercommissural distances; plots not shown). The correlation between tethering angles $(\mathrm{A} \alpha, \mathrm{S} \alpha$, and $\mathrm{P} \alpha)$ and preoperative TR severity, as well as tethering height on CT and preoperative TR severity, is shown in Figure 3, $D$ to $G$. Tethering angles and height correlated more strongly than TVADs with preoperative TR severity $\left(R^{2}\right.$ : $\mathrm{A} \alpha, 0.6810 ; \mathrm{S} \alpha, 0.5971 ;$ and $\mathrm{P} \alpha, 0.5769$; tethering height, $0.6854)$.

Regression plots of the postoperative TR jet area/RA area (continuous variable) of those who underwent TAP (22 patients) was associated with their tricuspid morphologic parameters and revealed that tethering angles and height significantly correlated with postoperative TR jet area/RA area (Figure 4). Demographic parameters that included age, sex, atrial fibrillation, pulmonary hypertension, and LV ejection fraction did not show significant correlation. Only tethering height remained as an independent risk factor on multivariate analysis $(P=.0069)$ (Table 3). 
A
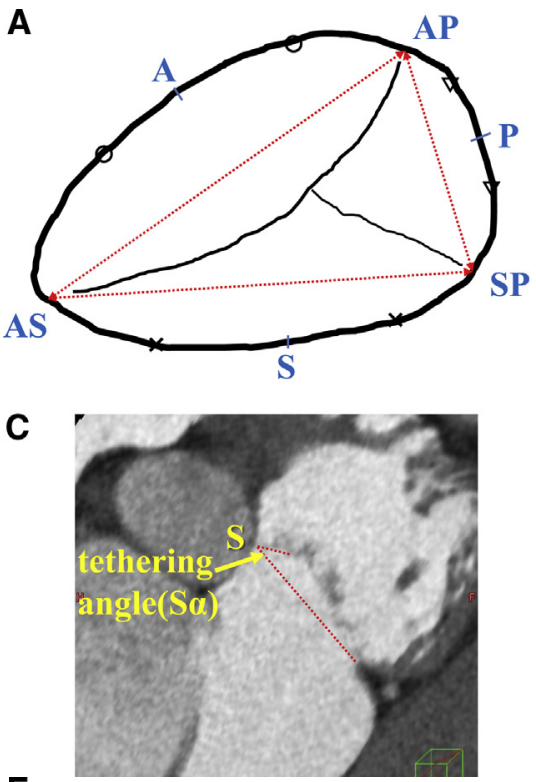

$\mathbf{E}$

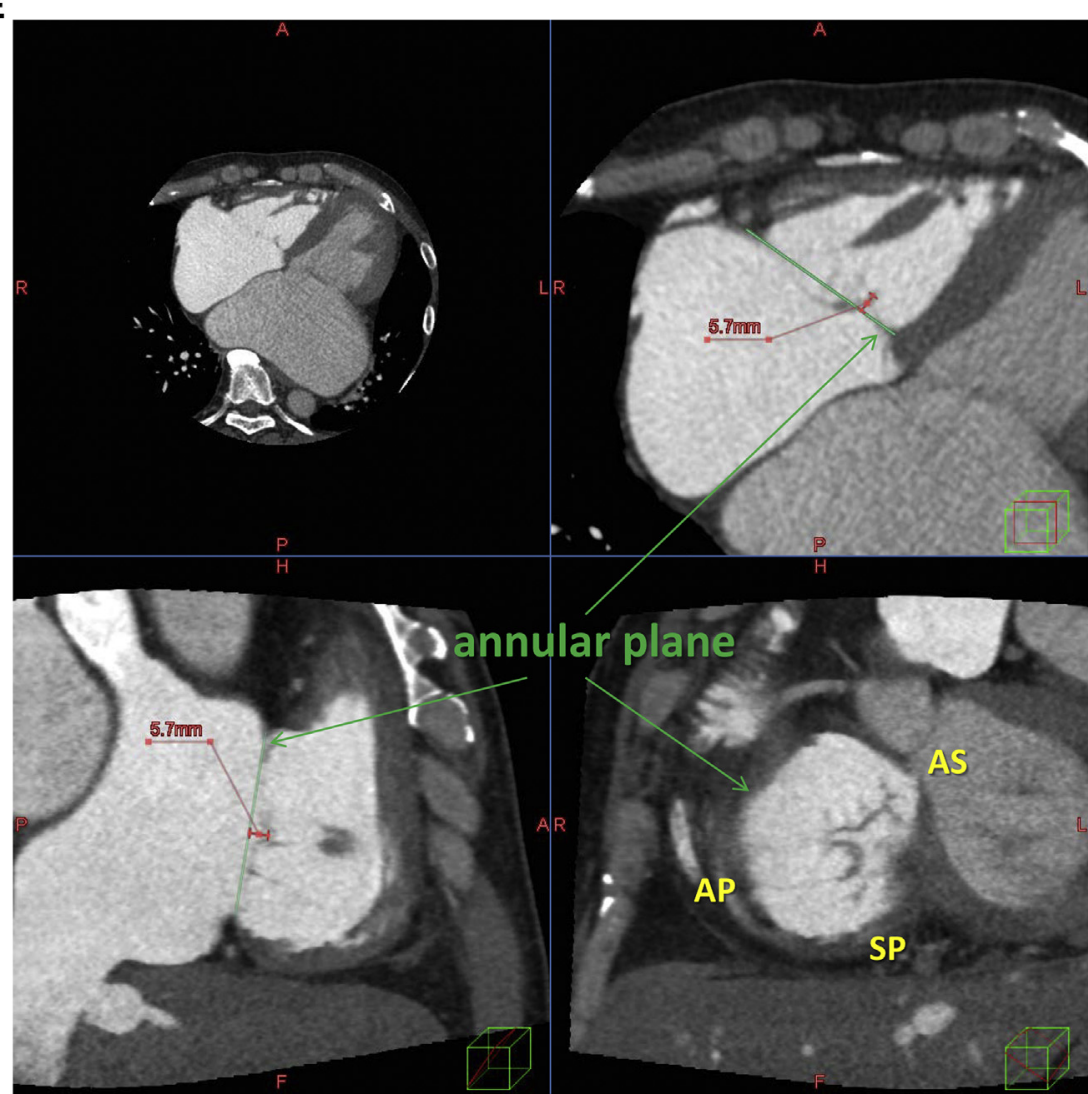

FIGURE 1. A, Scheme of the tricuspid valve and definition of each point along the annulus. $A$, Middle of anterior leaflet; $S$, middle of septal leaflet; $P$, middle of posterior leaflet; $A S$, anteroseptal commissure; $A P$, anteroposterior commissure; $S P$, septoposterior commissure. B and C, Actual measurement of the tricuspid valve annular diameter $(T V A D)$ and the tethering angles on computed tomography $(C T)$. D, Intraoperative measurement of distance between anteroseptal commissure and anteroposterior commissure with a sterile flexible ruler during tricuspid annuloplasty. E, Actual measurement of the tethering height of the tricuspid valve on CT. The maximum distance between the point of coaptation of the leaflets and the annular plane (defined as the plane containing $A S, A P$, and $S P$ ) was measured. 

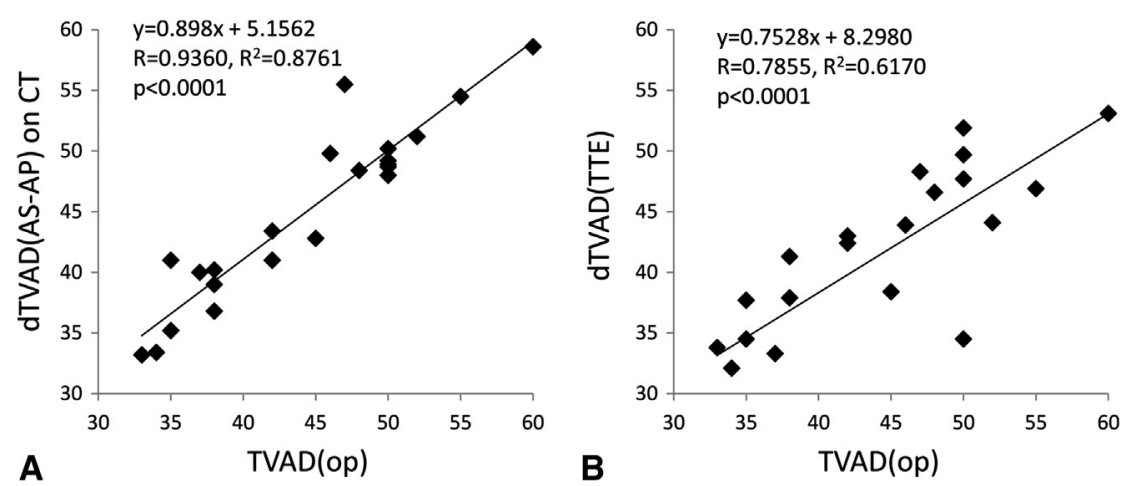

FIGURE 2. Regression plots between intraoperatively measured tricuspid valve annular diameter and end-diastolic tricuspid valve annular diameter examined by computed tomography (A) or 2-dimensional echocardiogram (B). TVAD(op), Tricuspid valve annular diameter measured during surgery; $d T V A$ $D(A S-A P)$, end-diastolic tricuspid valve annular diameter between anteroseptal commissure and anteroposterior commissure; $d T V A D(T T E)$, end-diastolic tricuspid valve annular diameter on transthoracic echocardiogram (TTE); $C T$, computed tomography.

Among the 22 patients who underwent TAP, 7 (31.8\%) patients had recurrent TR2, $1(4.5 \%)$ had TR3, and 1 $(4.5 \%)$ had TR4. Univariate and multivariate analyses determining the risk of developing recurrent TR2 or more revealed that preoperative tethering height on $\mathrm{CT}$ was a likely predictor (odds ratio, 2.838; 95\% confidence interval, 0.899-8.964; $P=.075$ ). Postoperative MR severity, LV diastolic diameter (LVDd), LV systolic diameter (LVDs), LV ejection fraction, and TR pressure gradient (TRPG) did not significantly correlate with postoperative TR severity $(P=.28, .090, .090, .12$, and .13 , respectively). No patient had significant pulmonary hypertension (TRPG $>31 \mathrm{~mm} \mathrm{Hg}$ ) postoperatively except for 1 patient (TRPG $44 \mathrm{~mm} \mathrm{Hg}$ ) who had only trivial postoperative TR. More than mild MR was found in 3 patients including 1 who underwent mitral valve repair for severe MR secondary to a severely dilated left ventricle owing to sarcoidosis and 1 who did not undergo mitral operation for MR that worsened after atrial septal defect closure. In these 3 patients with more than mild postoperative MR, TTE showed no postoperative TR.

A receiver operating characteristic curve was drawn to determine the cutoff value of the tethering height associated with recurrent TR. A tethering height of greater than 7.2 $\mathrm{mm}$ emerged as a likely cutoff value for the recurrence of TR2 or more after TAP (sensitivity, $72 \%$; specificity, $100 \%$ ).

\section{DISCUSSION}

To our knowledge, this is the first study to use ECG-gated 320-detector-row multislice CT for the measurement of the tricuspid valve apparatus. To validate the accuracy of the tricuspid valve measurements on $\mathrm{CT}$, we measured the TVADs on CT and 2-dimensional TTE and compared these with the intraoperative measurements of AS-AP. We realized that with the 2-dimensional TTE, the AS-AP distance was not easily measurable; in general, the annular diameter on TTE is represented by the maximum diastolic diameter obtainable from the 4-chamber view (dTVAD [TTE]). Despite the inconsistency in the measurement location, dTVAD(TTE) showed a favorable correlation with TVAD(op), although not as strongly as when dTVAD(AS-AP) on CT was correlated with TVAD(op). This may be due to an easier visualization and highly reproducible imaging of AS-AP on CT.

In the present study, we measured the geometric distances and angles of the tricuspid valve on CT and investigated their correlation with preoperative TR severity. Previous articles have described that tricuspid annular dilation mainly takes place at the segment corresponding to the free wall of right ventricle; thus, only the anterior and posterior annular segments, and rarely the septal segment, are responsible for the dilation. ${ }^{9,14,15}$ However, in this study, we discovered that all distances between each commissure elongated proportionally as the TR increased. This finding may explain why more recurrent $T R$ is observed after De Vega or flexible ring annuloplasties $5,13,16$ that repair only the anterior and posterior segments of the annulus. We also found that tethering angles and tethering height were more correlated than intercommissural distances with the preoperative severity of TR. Our findings imply that the severity of functional TR is more strongly determined by tethering rather than by annular dilation. The tethering height was measured in all leaflets, which did not show significant difference in our series. In case of severely distorted right ventricular configuration, the tethering height might be different in each leaflet and its identification may help to perform the repair with more consistency.

Various methods for determining the ring size for TAP have been adopted, such as measuring the actual diameter and downsizing it by two, ${ }^{8}$ using the same size ring as the mitral valve (for concomitant mitral and tricuspid valve procedures), ${ }^{17}$ choosing a fixed size for each gender (eg, $34 \mathrm{~mm}$ 

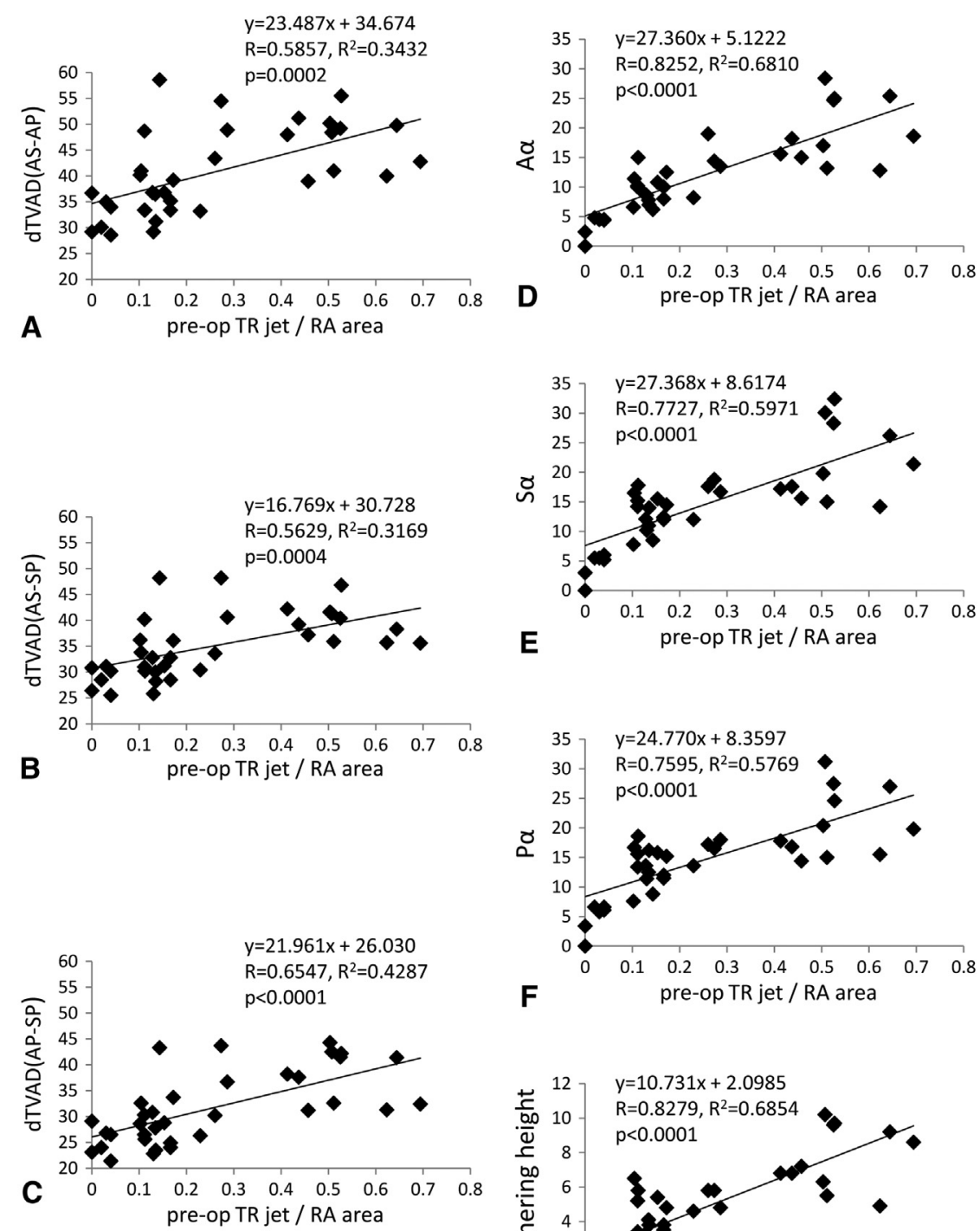

$\mathbf{F}$

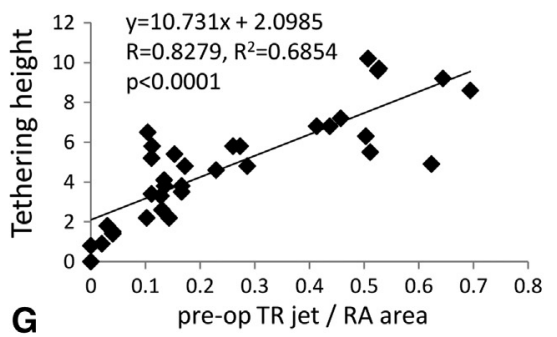

FIGURE 3. A to C, Regression plots between preoperative tricuspid regurgitation $(T R)$ severity and end-diastolic tricuspid valve annular diameter $(d T V A D)$ between each commissure. D to G, Regression plots between preoperative TR severity and the tethering angles or the tethering height. $d T V A D(A S-A P)$, Enddiastolic tricuspid valve annular diameter between anteroseptal commissure and anteroposterior commissure; $d T V A D(A S-S P)$, end-diastolic tricuspid valve annular diameter between anteroseptal commissure and septoposterior commissure; $d T V A D(A P-S P)$, end-diastolic tricuspid valve annular diameter between anteroposterior commissure and septoposterior commissure; $A \alpha$, tethering angle of anterior leaflet; $S \alpha$, tethering angle of septal leaflet; $P \alpha$, tethering angle of posterior leaflet; $R A$, right atrium.

for men and $32 \mathrm{~mm}$ for women), ${ }^{14}$ or basing it on the 3 quantiles of the human body mass (eg, $26 \mathrm{~mm}$ for small, $28 \mathrm{~mm}$ for normal, and $30 \mathrm{~mm}$ for large body mass). ${ }^{18,19}$ Provided with no absolute guideline as to what size ring to choose, most of the decisions are at the discretion of the surgeon. A review of the literature shows that TAP for functional TR, which included various ring types and sizes, may result in an early TR (TR3 or more) recurrence rate of between $14 \%$ and $16 \%{ }^{5,6}$ In our series of functional TR, we used a 28-mm ring (Edwards $\left.\mathrm{MC}^{3}\right)$ in all our patients except for 1 in whom we implanted a size 26 because of the small body mass, and our early TR (TR3 or more) recurrence rate was $9.1 \%$. We found in our preliminary study that dTVAD(AS-AP) on CT was about $30 \mathrm{~mm}$ in patients with no or trivial TR, and with this considered, the ring size that we have arbitrarily selected may have accounted for this improved outcome. It must be noted, as well, that only rigid rings were used in 

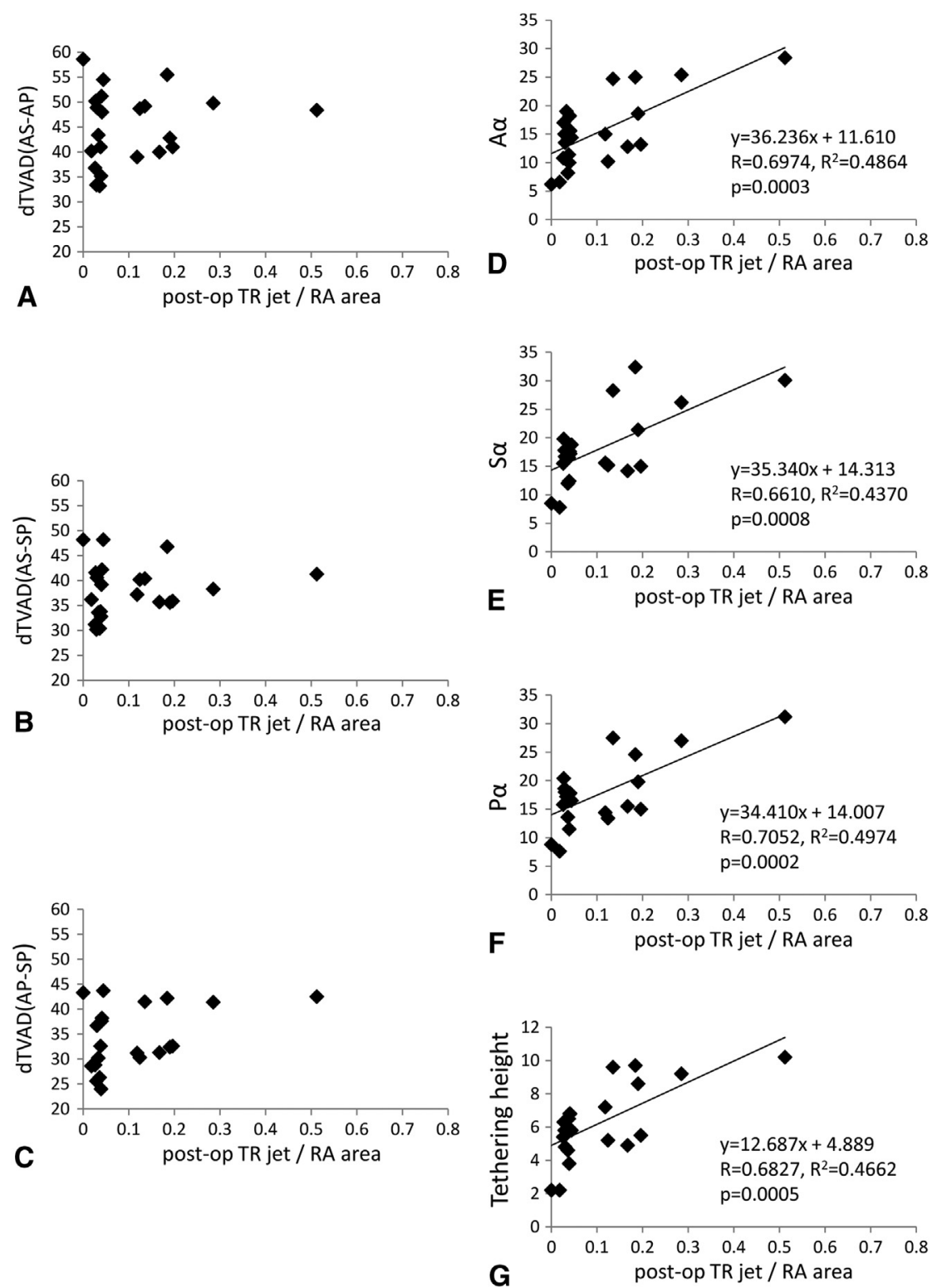

FIGURE 4. A to $\mathrm{C}$, Regression plots between postoperative tricuspid regurgitation $(T R)$ severity and end-diastolic tricuspid valve annular diameter ( $(T V A D)$ between each commissure. D to G, Regression plots between postoperative TR severity and the tethering angles or the tethering height. $d T V A D(A S-A P)$, End-diastolic tricuspid valve annular diameter between anteroseptal commissure and anteroposterior commissure; $d T V A D(A S-S P)$, enddiastolic tricuspid valve annular diameter between anteroseptal commissure and septoposterior commissure; $d T V A D(A P-S P)$, end-diastolic tricuspid valve annular diameter between anteroposterior commissure and septoposterior commissure; $d T V A D(A P-S P)$, end-diastolic tricuspid valve annular diameter between anteroposterior commissure and septoposterior commissure; $A \alpha$, tethering angle of anterior leaflet; $S \alpha$, tethering angle of septal leaflet; $P \alpha$, tethering angle of posterior leaflet; $R A$, right atrium.

this study. Our findings suggest that the entire tricuspid annulus can dilate, hence validating the speculation that if all surgeons had used an adequately sized rigid ring, their postoperative TR recurrence rates might have improved.

The etiology of the recurrence of TR after TAP remains unclear. Various risk factors have been identified, but lack consistency. Among these were age, low LV ejection fraction, preoperative TR severity, atrial fibrillation, preoperative permanent pacemaker, and pulmonary hypertension.
Some authors have indicated that the tethering distance and area, measured on a 2-dimensional echocardiogram, were also predictors of recurrent TR. ${ }^{5-7,18}$ Fukuda and associates, ${ }^{6}$ have also based their study on 2-dimensional echocardiographic findings and have reported that age, tethering distances of longer than $7.6 \mathrm{~mm}$, tethering area of greater than $1.63 \mathrm{~cm}^{2}$, and preoperative TR severity were significant predictors of recurrent TR. TVAD was not associated with the outcome of TAP in their study. From our risk 
TABLE 3. Univariate and multivariate risk factor analysis for TR jet area/RA area after TAP

\begin{tabular}{lc}
\hline & Univariate \\
\cline { 2 - 2 } & $\boldsymbol{P}$ value \\
\hline dTVAD(AS-AP) & .4885 \\
dTVAD(AS-SP) & .5273 \\
dTVAD(AP-SP) & .1556 \\
sTVAD(AS-AP) & .5652 \\
sTVAD(AS-SP) & .6596 \\
sTVAD(AP-SP) & .1098 \\
A $\alpha$ & .0003 \\
S $\alpha$ & .0008 \\
P $\alpha$ & .0002 \\
Tethering height & .0005 \\
age & .1358 \\
sex & .3399 \\
Af & .5315 \\
PH & .6245 \\
Low LVEF & .3076 \\
\hline
\end{tabular}

\begin{tabular}{lccc}
\hline & \multicolumn{3}{c}{ Multivariate } \\
\cline { 2 - 4 } & Degree of freedom & Mean square & $\boldsymbol{P}$ value \\
\hline dTVAD(AP-SP) & 1 & 0.015 & .1556 \\
Tethering height & 1 & 0.074 & .0069 \\
Age & 1 & 0.0020 & .1358 \\
\hline
\end{tabular}

TR jet area/RA area, Tricuspid regurgitation jet area over the area of the right atrium; $T A P$, tricuspid annuloplasty; TVAD, tricuspid valve annular diameter; $A S$, anteroseptal commissure; $A P$, anteroposterior commissure; $S P$, septoposterior commissure; $d$, end-diastolic; $s$, end-systolic; $A \alpha$, tethering angle of anterior leaflet; $S \alpha$, tethering angle of septal leaflet; $P \alpha$, tethering angle of posterior leaflet; $A f$, atrial fibrillation; $P H$, pulmonary hypertension; $L V E F$, left ventricular ejection fraction.

analysis using CT for data acquisition, tethering height emerged as an independent risk factor of recurrent TR. Furthermore, the receiver operating characteristic curve revealed that a tethering height of greater than $7.2 \mathrm{~mm}$ was the cutoff value for recurrent TR2 or more. Our findings are in congruous with the report by Fukuda and associates ${ }^{6}$ in that tethering of the leaflets was significantly correlated with recurrent TR whereas TVAD was not. Recently, Min and colleagues, ${ }^{13}$ using a 3-dimensional echocardiogram, have identified preoperative tenting volume and anteroposterior annulus diameter as independent predictors of residual TR severity. Their study, however, included patients who underwent various methods of tricuspid valve repair, including De Vega and flexible ring annuloplasty in $68 \%$ of their patients, which stresses the significance of procedural bias as a reason for the discordance in the results, particularly if given that the septal segment of the tricuspid annulus may elongate.

A tethering height of less than $7.2 \mathrm{~mm}$, as demonstrated in our study, could be the key factor for a successful tricuspid repair. In the operative setting, however, whether it be an arrested or a beating heart, measurement of the tethering height is not easy. Correcting the tethering height to its ideal length without a visible reference can be a problem, and confirming that it has been satisfactorily corrected is another. The TEE has been a valuable tool to assess valve structures and anatomy in this setting, but clear visualization of the tricuspid valve is often limited. Direct epicardial echocardiography might be a promising alternative in this respect inasmuch as it can be placed over the free wall of the right atrium and ventricle to obtain an unobstructed view, but its practicality and reliability need to be tested and reviewed.

In TAP, when ring implantation is applied, the TVAD may be reduced, but leaflet tethering may not be corrected. ${ }^{6,13}$ Ring annuloplasty itself may aggravate the tethering if the preoperative tethering is significant, and attempts to rectify this, such as by patch augmentation of the leaflets, ${ }^{20-22}$ or edge-to-edge repair (clover technique), ${ }^{23}$ or switching to a prosthetic valve replacement in some cases has previously been reported with varying outcomes. Our study has clearly demonstrated that the tricuspid valve apparatus can be geometrically measured. Perhaps by tailoring and optimizing the procedures to individual morphologic differences identifiable on $\mathrm{CT}$ or other diagnostic modalities, the outcomes may reach consistency. Further studies, however, are warranted.

It has been speculated that tricuspid leaflet tethering occurs secondary to papillary muscle displacement caused by changes in volume and/or geometry of the right ventricle. ${ }^{13}$ A thorough assessment of the right ventricular anatomy may therefore offer us additional insight into the mechanism of functional TR and its recurrence. Recently, an article reporting on right ventricular papillary muscle displacement in patients with a dilated left ventricle and significant TR was published. ${ }^{24}$ The application of the 320-detector-row multislice CT, particularly for a detailed assessment of the intraventricular structures, may be one attractive option to facilitate adequately tailored surgical repair and improved long-term outcome for these group of patients.

\section{Study Limitations}

This study has a number of limitations. Some of the major ones include the retrospective design, the small number of patients, and the single-center study. A prospective study consisting of a larger patient cohort is essential to verify the reproducibility of the results obtained from multivariate analysis and to validate the cutoff value determined in this study.

Another limitation is the study period. Long-term results need to be clarified, inasmuch as TR can progress with time. ${ }^{5,8,16}$ A longer follow-up period may potentially change the results of our study.

The tricuspid valve structure was not evaluated postoperatively because we have not found a way to reduce the halation caused by the prosthetic ring on CT. It is unclear whether recurrent TR may be affected by changes, if any, in post-TAP tricuspid valve structure. 
Finally, the potential pathologic influence of the right atrial and ventricular function was not addressed in the present study. The severity of TR may be affected by variables such as preload, afterload, and dilation of the right chambers that can confound our results. Further studies should aim at looking into all these issues.

\section{CONCLUSIONS}

TVADs, tethering angles, and tethering height correlated with preoperative TR severity. Tethering angles and tethering height was significantly correlated with recurrent TR whereas TVAD was not. On multivariate analysis, tethering height emerged as an independent risk factor for postoperative TR. For patients in whom postoperative TR2 or more developed, tethering height remained as a likely predictor.

We thank Yasunori Sato, PhD (Clinical Research Center, Graduate School of Medicine, Chiba University, Chiba, Japan) for his assistance with the statistical analysis.

\section{References}

1. Shiran A, Sagie A. Tricuspid regurgitation in mitral valve disease: incidence, prognostic implications, mechanism, and management. J Am Coll Cardiol. 2009;53:401-8.

2. Rogers JH, Bolling SF. The tricuspid valve: current perspective and evolving management of tricuspid regurgitation. Circulation. 2009;119:2718-25.

3. Nath J, Foster E, Heidenreich PA. Impact of tricuspid regurgitation on long-term survival. J Am Coll Cardiol. 2004;43:405-9.

4. Tang GH, David TE, Singh SK, Maganti MD, Armstrong S, Borger MA. Tricuspid valve repair with an annuloplasty ring results in improved long-term outcomes. Circulation. 2006;14(1 Suppl):I577-81

5. McCarthy PM, Bhudia SK, Rajeswaran J, Hoercher KJ, Lytle BW, Cosgrove DM, et al. Tricuspid valve repair: Durability and risk factors for failure. J Thorac Cardiovasc Surg. 2004;127:674-85.

6. Fukuda S, Song JM, Gillinov M, McCarthy PM, Daimon M, Kongsaerepong V, et al. Tricuspid valve tethering predicts residual tricuspid regurgitation after tricuspid annuloplasty. Circulation. 2005;111:975-9.

7. Fukuda S, Gillinov M, McCarthy PM, Matsumura Y, Thomas JD, Shiota T. Echocardiographic follow-up of tricuspid annuloplasty with a new three-dimensional ring in patients with functional tricuspid regurgitation. J Am Soc Echocardiogr. 2007;20:1236-42.

8. Sales V, McCarthy PM. Durability of functional tricuspid valve repair. Semin Thorac Cardiovasc Surg. 2010;22:97-103.

9. Ton-Nu TT, Levine RA, Handschumacher MD, Dorer DJ, Yosefy C, Fan D, et al. Geometric determinants of functional tricuspid regurgitation: insights from 3-dimensional echocardiography. Circulation. 2006;114:143-9.
10. Fukuda S, Saracino G, Matsumura Y, Daimon M, Tran H, Greenberg NL, et al. Three-dimensional geometry of the tricuspid annulus in healthy subjects and in patients with functional tricuspid regurgitation: a real-time, 3-dimensional echocardiographic study. Circulation. 2006;14(Suppl I):I492-8.

11. Sukmawan R, Watanabe M, Ogasawara Y, Yamaura Y, Yamamoto K, Wada N, et al. Geometric changes of tricuspid valve tenting in tricuspid regurgitation secondary to pulmonary hypertension quantified by novel system with transthoracic real-time 3-dimensional echocardiography. J Am Soc Echocardiogr. 2007;20: 470-6.

12. Park YH, Song JM, Lee EY, Kim YJ, Kang DH, Song JK. Geometric and hemodynamic determinants of functional tricuspid regurgitation: a real-time three-dimensional echocardiography study. Int J Cardiol. 2008;124:160-5.

13. Min SY, Song JM, Kim JH, Jang MK, Kim YJ, Song H, et al. Geometric changes after tricuspid annuloplasty and predictors of residual tricuspid regurgitation: a real-time three-dimensional echocardiography study. Eur Heart J. 2010;31: 2871-80.

14. Dreyfus GD, Corbi PJ, John KM, Bahrami T. Secondary tricuspid regurgitation or dilatation: which should be the criteria for surgical repair? Ann Thorac Surg. 2005;79:127-32.

15. Chan KMJ, Zakkar M, Amirak E, Punjabi PP. Tricuspid valve disease: pathophysiology and optimal management. Prog Cardiovasc Dis. 2009;51: 482-6.

16. Navia JL, Nowiki ER, Blackstone EH, Brozzi NA, Nento DE, Atik FA, et al. Surgical management of secondary tricuspid valve regurgitation: annulus, commissure, or leaflet procedure? J Thorac Cardiovasc Surg. 2010;139: 1473-82.

17. Rogers JH, Bolling SH. Valve repair for functional tricuspid valve regurgitation: anatomical and surgical considerations. Semin Thoracic Surg. 2010;22: 84-9.

18. Fukuda S, Gillinov M, McCarthy PM, Stewart WJ, Song JM, Kihara T, et al. Determinants of recurrent or residual functional tricuspid regurgitation after tricuspid annuloplasty. Circulation. 2006;14(Suppl):I582-7.

19. Ghoreishi M, Brown JM, Stauffer CE, Young CA, Byron MJ, Griffith BP, et al. Undersized tricuspid annuloplasty rings optimally treat functional tricuspid regurgitation. Ann Thorac Surg. 2011;92:89-96.

20. Choi JB, Kim NY, Kim KH, Kim MH, Jo JK. Tricuspid leaflet augmentation to eliminate residual regurgitation in severe functional tricuspid regurgitation. Ann Thorac Surg. 2011;92:e131-3.

21. Dreyfus GD, Raja SG, Chan KMJ. Tricuspid leaflet augmentation to address severe tethering in functional tricuspid regurgitation. Eur J Cardiothorac Surg. 2008;34:908-10.

22. Roshanali F, Saidi B, Mandegar MH, Yousefina MA, Alaeddini F. Echocardiographic approach to the decision-making process for tricuspid valve repair. J Thorac Cardiovasc Surg. 2010;139:1483-7.

23. Lapenna E, Bonis MD, Verzini A, Canna GL, Ferrara D, Calabrese MC, et al. The clover technique for the treatment of complex tricuspid valve insufficiency: midterm clinical and echocardiographic results in 66 patients. Eur J Cardiothorac Surg. 2010;37:1297-303.

24. Spinner EM, Sundareswaran K, Dasi LP, Thourani VH, Oshinski J, Yoganathan AP. Altered right ventricular papillary muscle position orientation in patients with a dilated left ventricle. J Thorac Cardiovasc Surg. 2011;141: 744-9. 